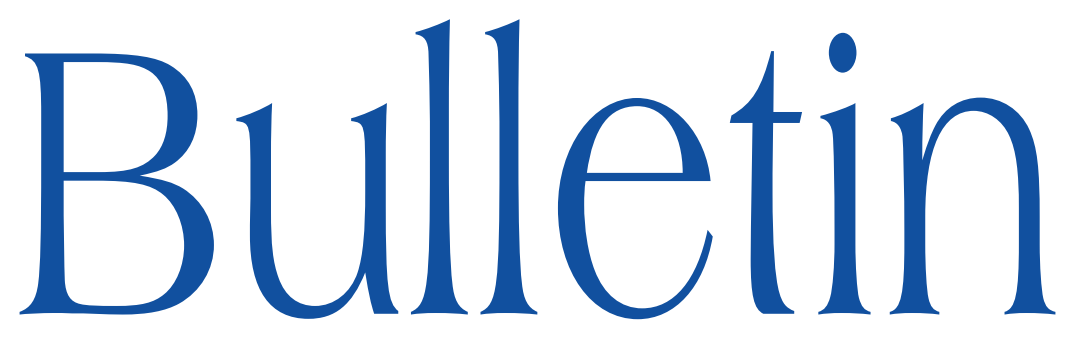

de la SOCIÉTÉ MATHÉMATIQUE DE FRANCE

\title{
ON A CERTAIN GENERALIZATION OF SPHERICAL TWISTS
}

\author{
Yukinobu Toda
}

Tome 135

Fascicule 1

2007 


\title{
ON A CERTAIN GENERALIZATION OF SPHERICAL TWISTS
}

\author{
BY YUKINOBU TODA
}

\begin{abstract}
This note gives a generalization of spherical twists, and describe the autoequivalences associated to certain non-spherical objects. Typically these are obtained by deforming the structure sheaves of $(0,-2)$-curves on threefolds, or deforming $\mathbb{P}$-objects introduced by D. Huybrechts and R. Thomas.
\end{abstract}

RÉSumé (Sur une généralisation des twists sphériques). - Cette note donne une généralisation des twists sphériques et décrit des auto-équivalences associées à certains objets qui ne sont pas sphériques. Typiquement ces objets sont obtenus par déformation du faisceau structural d'une $(0,2)$-courbe dans une variété de dimension trois ou d'un $\mathbb{P}$-objet introduit par D. Huybrechts et R. Thomas.

\section{Introduction}

We introduce a new class of autoequivalences of derived categories of coherent sheaves on smooth projective varieties, which generalizes the notion of spherical twists given in [12]. Such autoequivalences are associated to a certain class of objects, which are not necessary spherical but are interpreted as "fat"

Texte reçu le 4 avril 2006, révisé le 23 mai 2006

Yukinobu TodA, Yukinobu Toda, Graduate School of Mathematical Sciences, University of Tokyo, 3-1-8 Komaba, Meguro, Tokyo 153-8914 (Japan)

E-mail : toda@ms.u-tokyo.ac.jp

2000 Mathematics Subject Classification. - 18E30, 14J32.

Key words and phrases. — Derived categories, mirror symmetries. 
version of them. We introduce the notion of $R$-spherical objects for a noetherian and artinian local $\mathbb{C}$-algebra $R$, and imitate the construction of spherical twists to give the associated autoequivalences.

Let $X$ be a smooth complex projective variety, and $D(X)$ be a bounded derived category of coherent sheaves on $X$. When $X$ is a Calabi-Yau 3-fold, $D(X)$ is considered to represent the category of $D$-branes of type $B$, and should be equivalent to the derived Fukaya category on a mirror manifold under Homological mirror symmetry [8]. On the mirror side, there are typical symplectic automorphisms by taking Dehn twists along Lagrangian spheres [11]. The notions of spherical objects and associated twists were introduced in [12] in order to realize Dehn twists under mirror symmetry. Recall that $E \in D(X)$ is called spherical if the following holds [12]:

- $\operatorname{Ext}_{X}^{i}(E, E)= \begin{cases}\mathbb{C} & \text { if } i=0 \text { or } i=\operatorname{dim} X, \\ 0 & \text { otherwise; }\end{cases}$

- $E \otimes \omega_{X} \cong E$.

Then one can construct the autoequivalence $T_{E}: D(X) \rightarrow D(X)$ which fits into the distinguished triangle [12]:

$$
\mathbb{R} \operatorname{Hom}(E, F) \otimes_{\mathbb{C}} E \longrightarrow F \longrightarrow T_{E}(F),
$$

for $F \in D(X)$. The autoequivalence $T_{E}$ is called a spherical twist. This is a particularly important class of autoequivalences, especially when we consider $A_{n}$-configulations on surfaces as indicated in [7]. On the other hand, it has been observed that there are some autoequivalences which are not described in terms of spherical twists. This occurs even in the similar situation discussed in [7] as follows. Let $X \rightarrow Y$ be a three dimensional flopping contraction which contracts a rational curve $C \subset X$, and $X^{\dagger} \rightarrow Y$ be its flop. Then one can construct the autoequivalence $[1,3,4]$,

$$
\Phi:=\Phi_{X^{\dagger} \rightarrow X}^{\mathcal{O}_{X X^{\prime} X^{\dagger}}} \circ \Phi_{X \rightarrow X^{\dagger}}^{\mathcal{O}_{X X_{Y} X^{\dagger}}}: D(X) \longrightarrow D\left(X^{\dagger}\right) \longrightarrow D(X) .
$$

If $C \subset X$ is not a $(-1,-1)$-curve, $\Phi$ is not written as a spherical twist, and our motivation comes from describing such autoequivalences. Let $R$ be a noetherian and artinian local $\mathbb{C}$-algebra. We introduce the notion of $R$-spherical objects defined on $D(X \times \operatorname{Spec} R)$. In the above example, $\operatorname{Spec} R$ is taken to be the moduli space of $\mathcal{O}_{C}(-1)$, and the universal family gives the $R$-spherical object. Our main theorem is the following:

TheOREM 1.1. - To any $R$-spherical object $\mathcal{E} \in D(X \times \operatorname{Spec} R)$, we can associate the autoequivalence $T_{\mathcal{E}}: D(X) \rightarrow D(X)$, which fits into the distinguished triangle

$$
\mathbb{R} \operatorname{Hom}_{X}\left(\pi_{*} \mathcal{E}, F\right) \stackrel{\mathbb{L}}{\otimes_{R}} \pi_{*} \mathcal{E} \longrightarrow F \longrightarrow T_{\mathcal{E}}(F),
$$


for $F \in D(X)$. Here $\pi: X \times \operatorname{Spec} R \rightarrow X$ is the projection.

Using the notion of $R$-spherical objects and associated twists, we can also give the deformations of $\mathbb{P}$-twists in the case which is not treated in [5].

Acknowledgement. - The author thanks Tom Bridgeland for useful discussions and comments. He is supported by Japan Society for the Promotion of Sciences Research Fellowships for Young Scientists, No 1611452.

\section{Notations and conventions}

- For a variety $X$, we denote by $D(X)$ its bounded derived category of coherent sheaves.

- $\Delta$ means the diagonal $\Delta \subset X \times X$ or the diagonal embedding $\Delta: X \rightarrow$ $X \times X$.

- For another variety $Y$ and an object $\mathcal{P} \in D(X \times Y)$, denote by $\Phi_{X \rightarrow Y}^{\mathcal{P}}$ the integral transform with kernel $\mathcal{P}$, i.e.,

$$
\Phi_{X \rightarrow Y}^{\mathcal{P}}(*):=\mathbb{R} p_{Y *}\left(p_{X}^{*}(*) \stackrel{\mathbb{L}}{\otimes} \mathcal{P}\right): D(X) \longrightarrow D(Y) .
$$

Here $p_{X}, p_{Y}$ are projections from $X \times Y$ onto corresponding factors.

\section{Generalized spherical twists}

Let $X$ be a smooth projective variety over $\mathbb{C}$ and $R$ be a noetherian and artinian local $\mathbb{C}$-algebra. We introduce the notion of $R$-spherical objects defined on $D(X \times \operatorname{Spec} R)$. Let $\pi: X \times \operatorname{Spec} R \rightarrow X$ and $\pi^{\prime}: X \times \operatorname{Spec} R \rightarrow \operatorname{Spec} R$ be projections and $0 \in \operatorname{Spec} R$ be the closed point.

Definition 2.1. - An object $\mathcal{E} \in D(X \times \operatorname{Spec} R)$ is called $R$-spherical if the following conditions hold:

- $\mathcal{E}$ is represented by a bounded complex $\mathcal{E}^{\bullet}$ with each $\mathcal{E}^{i}$ a coherent

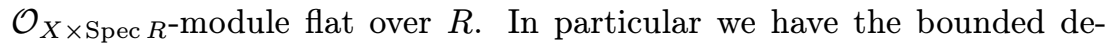
rived restriction $E:=\left.\mathcal{E}^{\bullet}\right|_{X \times\{0\}} \in D(X)$.

- $\operatorname{Ext}_{X}^{i}(E, E)= \begin{cases}\mathbb{C} & \text { if } i=0 \text { or } i=\operatorname{dim} X, \\ 0 & \text { otherwise; }\end{cases}$

- $E \otimes \omega_{X} \cong E$.

REMARK 2.2. - If $R=\mathbb{C}$, then $R$-spherical objects coincide with usual spherical objects.

We imitate the construction of the spherical twists in the following theorem. 
TheOREM 2.3. - To any $R$-spherical object $\mathcal{E} \in D(X \times \operatorname{Spec} R)$, we can associate the autoequivalence $T_{\mathcal{E}}: D(X) \rightarrow D(X)$, which fits into the distinguished triangle:

$$
\mathbb{R} \operatorname{Hom}_{X}\left(\pi_{*} \mathcal{E}, F\right) \stackrel{\mathbb{L}}{\otimes_{R}} \pi_{*} \mathcal{E} \longrightarrow F \longrightarrow T_{\mathcal{E}}(F),
$$

for $F \in D(X)$. Here $R$-module structures on $\mathbb{R} \operatorname{Hom}_{X}\left(\pi_{*} \mathcal{E}, F\right)$ and $\pi_{*} \mathcal{E}$ are inherited from $R$-module structure on $\mathcal{E}$.

Proof. - First we construct the kernel of $T_{\mathcal{E}}$. Let $p_{i j}$ and $p_{i}$ be projections as in the following diagram

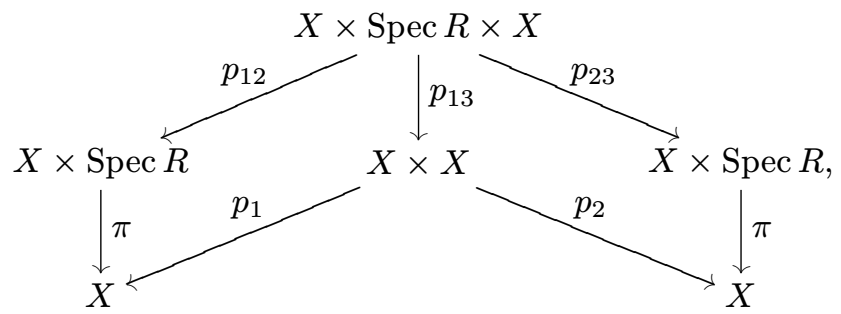

and consider the object

$$
\mathcal{Q}:=\mathbb{R} p_{13 *}\left(p_{12}^{*}\left(\pi^{!} \mathcal{O}_{X} \stackrel{\mathbb{L}}{\otimes} \check{\mathcal{E}}\right) \stackrel{\mathbb{E}}{\otimes} p_{23}^{*} \mathcal{E}\right) \in D(X \times X) .
$$

Here $\check{\mathcal{E}}$ means its derived dual. Then for $F \in D(X)$, we can calculate $\Phi_{X \rightarrow X}^{\mathcal{Q}}(F)$ as follows:

$$
\begin{aligned}
\Phi_{X \rightarrow X}^{\mathcal{O}}(F) & \cong \mathbb{R} p_{2 *}\left(\mathbb{R} p_{13 *}\left(p_{12}^{*}\left(\pi^{!} \mathcal{O}_{X} \stackrel{\mathbb{L}}{\otimes} \check{\mathcal{E}}\right) \stackrel{\mathbb{L}}{\otimes} p_{23}^{*} \mathcal{E}\right) \stackrel{\mathbb{L}}{\otimes} p_{1}^{*} F\right) \\
& \cong \mathbb{R} p_{2 *} \mathbb{R} p_{13 *}\left(p_{12}^{*}\left(\pi^{!} \mathcal{O}_{X} \stackrel{\mathbb{L}}{\otimes} \check{\mathcal{E}}\right) \stackrel{\mathbb{L}}{\otimes} p_{23}^{*} \mathcal{E} \stackrel{\mathbb{L}}{\otimes} p_{13}^{*} p_{1}^{*} F\right) \\
& \cong \pi_{*} \mathbb{R} p_{23 *}\left(p_{12}^{*}\left(\pi^{!} \mathcal{O}_{X} \stackrel{\mathbb{L}}{\otimes} \check{\mathcal{E}}\right) \stackrel{\mathbb{L}}{\otimes} p_{23}^{*} \mathcal{E} \stackrel{\mathbb{L}}{\otimes} p_{12}^{*} \pi^{*} F\right) \\
& \cong \pi_{*}\left\{\mathcal{E} \stackrel{\mathbb{E}}{\otimes} \mathbb{R} p_{23 *} p_{12}^{*}\left(\pi^{!} \mathcal{O}_{X} \stackrel{\mathbb{L}}{\otimes} \check{\mathcal{E}} \otimes \pi^{*} F\right)\right\} \\
& \cong \pi_{*}\left\{\mathcal{E} \stackrel{\mathbb{L}}{\otimes} \pi^{*} \mathbb{R} \pi_{*}^{\prime} \mathbb{R} \mathcal{H} \text { om }\left(\mathcal{E}, \pi^{!} F\right)\right\} \\
& \cong \pi_{*} \mathcal{E} \stackrel{\mathbb{L}}{\otimes}_{R} \mathbb{R} \operatorname{Hom}\left(\pi_{*} \mathcal{E}, F\right) .
\end{aligned}
$$

The fifth equality comes from the base change formula for the diagram below:

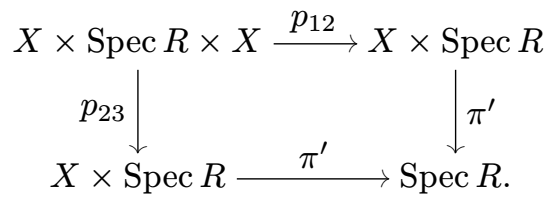

TOME $135-2007-\mathrm{N}^{\mathrm{O}} 1$ 
On the other hand, we have

$$
\begin{aligned}
\operatorname{Hom}_{X \times X}\left(\mathcal{Q}, \mathcal{O}_{\Delta}\right) & =\operatorname{Hom}_{X \times X}\left(\mathbb{R} p_{13 *}\left(p_{12}^{*}\left(\pi^{!} \mathcal{O}_{X} \stackrel{\mathbb{L}}{\otimes} \check{\mathcal{E}}\right) \stackrel{\mathbb{L}}{\otimes} p_{23}^{*} \mathcal{E}\right), \mathcal{O}_{\Delta}\right) \\
& =\operatorname{Hom}_{X}\left(\mathbb{L} \Delta^{*} \mathbb{R} p_{13 *}\left(p_{12}^{*}\left(\pi^{!} \mathcal{O}_{X} \stackrel{\mathbb{L}}{\otimes} \check{\mathcal{E}}\right) \stackrel{\mathbb{L}}{\otimes} p_{23}^{*} \mathcal{E}\right), \mathcal{O}_{X}\right) \\
& =\operatorname{Hom}_{X}\left(\pi_{*} \mathbb{L}(\Delta, \mathrm{id})^{*}\left(p_{12}^{*}\left(\pi^{!} \mathcal{O}_{X} \stackrel{\mathbb{L}}{\otimes} \check{\mathcal{E}}\right) \stackrel{\mathbb{L}}{\otimes} p_{23}^{*} \mathcal{E}\right), \mathcal{O}_{X}\right) \\
& =\operatorname{Hom}_{X}\left(\mathbb{L}(\Delta, \mathrm{id})^{*}\left(p_{12}^{*}\left(\pi^{!} \mathcal{O}_{X} \stackrel{\mathbb{L}}{\otimes} \check{\mathcal{E}}\right) \stackrel{\mathbb{L}}{\otimes} p_{23}^{*} \mathcal{E}\right), \pi^{!} \mathcal{O}_{X}\right) \\
& =\operatorname{Hom}_{X}\left(\pi^{!} \mathcal{O}_{X} \stackrel{\mathbb{L}}{\otimes} \check{\mathcal{E}} \stackrel{\mathbb{L}}{\otimes} \mathcal{E}, \pi^{!} \mathcal{O}_{X}\right) .
\end{aligned}
$$

The third equality comes from the base change formula for the diagram below:

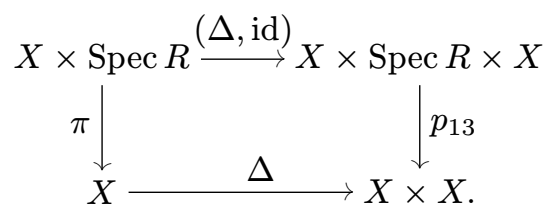

Let $\mu: \mathcal{Q} \rightarrow \mathcal{O}_{\Delta}$ be the morphism which corresponds to the morphism

$$
\operatorname{id}_{\pi^{\prime} \mathcal{O}_{X}} \otimes \mathrm{ev}: \pi^{!} \mathcal{O}_{X} \stackrel{\mathbb{L}}{\otimes} \check{\mathcal{E}} \stackrel{\mathbb{L}}{\otimes} \mathcal{E} \longrightarrow \pi^{!} \mathcal{O}_{X}
$$

under the above isomorphisms. Let us take its cone $\mathcal{R}:=\operatorname{Cone}(\mu) \in D(X \times X)$. Then the above calculation for $\Phi_{X \rightarrow X}^{\mathcal{Q}}$ implies the functor $T_{\mathcal{E}}: D(X) \rightarrow D(X)$ with kernel $\mathcal{R}$ fits into the triangle

$$
\mathbb{R} \operatorname{Hom}_{X}\left(\pi_{*} \mathcal{E}, F\right) \stackrel{\mathbb{L}}{\otimes_{R}} \pi_{*} \mathcal{E} \longrightarrow F \longrightarrow T_{\mathcal{E}}(F),
$$

for $F \in D(X)$. We check $T_{\mathcal{E}}$ gives an equivalence. We follow the arguments of $[10,5]$. Define $E^{\perp}$ to be the subcategory $\{F \in D(X) \mid \mathbb{R} \operatorname{Hom}(E, F)=0\}$. Then $\Omega:=E \cup E^{\perp}$ is a spanning class in the sense of [2, Def. 2.1]. Let $\langle E\rangle$ be the minimum extension closed subcategory of $D(X)$ which contains $E$. Then since $R$ is finite dimensional, we have $\pi_{*} \mathcal{E} \in\langle E\rangle$. Therefore if $F \in E^{\perp}$, then $\mathbb{R} \operatorname{Hom}_{X}\left(\pi_{*} \mathcal{E}, F\right)=0$. Hence $T_{\mathcal{E}}(F) \cong F$ for $F \in E^{\perp}$. Next since $\mathcal{E}$ is $R$ spherical, we have the distinguished triangle

$$
E \longrightarrow \mathbb{R} \operatorname{Hom}_{X}\left(\pi_{*} \mathcal{E}, E\right) \stackrel{\mathbb{L}}{\otimes_{R}} \pi_{*} \mathcal{E} \longrightarrow E[-\operatorname{dim} X]
$$


Then the following diagram

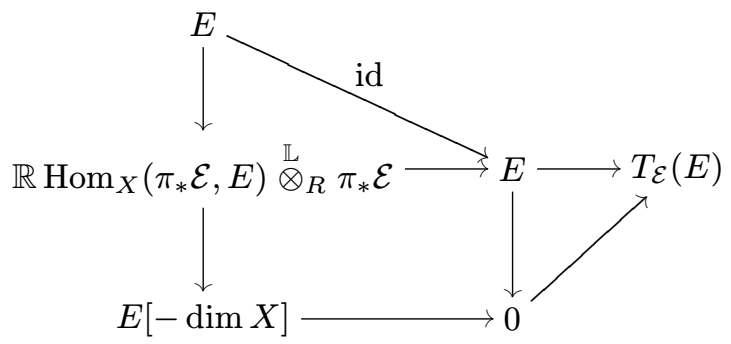

shows $T_{\mathcal{E}}(E) \cong E[1-\operatorname{dim} X]$. Therefore $T_{\mathcal{E}}$ is fully faithful on $\Omega$, hence fully faithful on $D(X)$. (cf. [2, Theorem 2.3]). Finally the assumption $E \otimes \omega_{X} \cong E$ implies $F \otimes \omega_{X} \in E^{\perp}$ for $F \in E^{\perp}$. Therefore $\left.T_{\mathcal{E}}\right|_{\Omega}$ commutes with $\otimes \omega_{X}$. Hence $T_{\mathcal{E}}$ gives an equivalence by the argument of [2, Thm 5.4].

\section{Flops at $(0,-2)$-curves}

We give some examples of autoequivalences associated to $R$-spherical objects. Let $f: X \rightarrow Y$ be a three dimensional flopping contraction which contracts a rational curve $C \subset X$. Let $f^{\dagger}: X^{\dagger} \rightarrow Y$ be its flop, and $C^{\dagger} \subset X^{\dagger}$ be the flopped curve. Then in [1,3,4], the functor $\Phi_{1}: D\left(X^{\dagger}\right) \rightarrow D(X)$ with kernel $\mathcal{O}_{X \times_{Y} X^{\dagger}}$ gives an equivalence. $\Phi_{1}$ satisfies the following (cf. [14, Lemma 5.1]):

- $\Phi_{1}$ takes $\mathcal{O}_{C^{\dagger}}(-1)[1]$ to $\mathcal{O}_{C}(-1)$;

- $\Phi_{1}$ commutes with derived push-forwards, i.e., $\mathbb{R} f_{*} \circ \Phi_{1} \cong \mathbb{R} f_{*}^{\dagger}$.

Similarly we can construct the equivalence $\Phi_{2}: D(X) \rightarrow D\left(X^{\dagger}\right)$ with kernel $\mathcal{O}_{X \times_{Y} X^{\dagger}}$. Composing these, we obtain the autoequivalence

$$
\Phi:=\Phi_{1} \circ \Phi_{2}: D(X) \longrightarrow D\left(X^{\dagger}\right) \longrightarrow D(X) .
$$

Note that $\Phi\left(\mathcal{O}_{C}(-1)\right)=\mathcal{O}_{C}(-1)[-2]$ and $\Phi$ commutes with $\mathbb{R} f_{*}$. If $C \subset X$ is a $(-1,-1)$-curve, then $\mathcal{O}_{C}(-1)$ is a spherical object and $\Phi$ coincides with the associated twist $T_{\mathcal{O}_{C}(-1)}$. But if $C$ is not a $(-1,-1)$-curve, then $\mathcal{O}_{C}(-1)$ is no longer spherical, so we have to find some new descriptions of $\Phi$. The idea is to consider the moduli problem of $\mathcal{O}_{C}(-1)$ and use the universal family.

Here we assume $C \subset X$ is a $(0,-2)$-curve, i.e., normal bundle is $\mathcal{O}_{C} \oplus$ $\mathcal{O}_{C}(-2)$, and give the description of $\Phi$. Let $\mathcal{M}$ be the connected component of the moduli space of simple sheaves on $X$, which contains $\mathcal{O}_{C}(-1)$. We define

$$
R_{m}:=\mathbb{C}[t] /\left(t^{m+1}\right), \quad S_{m}:=\operatorname{Spec} R_{m} .
$$

Since $\operatorname{Ext}_{X}^{1}\left(\mathcal{O}_{C}(-1), \mathcal{O}_{C}(-1)\right)=\mathbb{C}$ and $C \subset X$ is rigid, we can write $\mathcal{M}$ as $\mathcal{M}=S_{m}$ for some $m \in \mathbb{N}$. Let $\mathcal{E} \in \operatorname{Coh}\left(X \times S_{m}\right)$ be the universal family. 
TheOREm 3.1. - $\mathcal{E}$ is a $R_{m}$-spherical object and the associated functor

$$
T_{\mathcal{E}}: D(X) \longrightarrow D(X)
$$

coincides with $\Phi$.

Proof. - For $n \leq m$, define $\mathcal{E}_{n}$ to be

$$
\mathcal{E}_{n}:=\pi_{n *}\left(\left.\mathcal{E}\right|_{X \times S_{n}}\right) \in \operatorname{Coh}(X),
$$

where $\pi_{n}: X \times S_{n} \rightarrow X$ is a projection. Since we have the exact sequences of $R_{m}$-modules

$$
\begin{aligned}
& 0 \longrightarrow R_{n-1} \longrightarrow R_{n} \longrightarrow \mathbb{C} \longrightarrow 0 \\
& 0 \longrightarrow \mathbb{C} \longrightarrow R_{n} \longrightarrow R_{n-1} \longrightarrow 0
\end{aligned}
$$

we have the exact sequences in $\operatorname{Coh}(X)$ :

$$
\begin{aligned}
& 0 \longrightarrow \mathcal{E}_{n-1} \longrightarrow \mathcal{E}_{n} \longrightarrow E \longrightarrow 0, \\
& 0 \longrightarrow E \longrightarrow \mathcal{E}_{n} \longrightarrow \mathcal{E}_{n-1} \longrightarrow 0
\end{aligned}
$$

Here $E:=\mathcal{O}_{C}(-1)$. Applying $\operatorname{Hom}(*, E)$ to the sequence (1), we obtain the long exact sequence

$$
\begin{aligned}
\operatorname{Hom}\left(\mathcal{E}_{n}, E\right) & \longrightarrow \operatorname{Hom}\left(\mathcal{E}_{n-1}, E\right) \stackrel{\xi_{n}}{\longrightarrow} \operatorname{Ext}^{1}(E, E)=\mathbb{C} \\
& \longrightarrow \operatorname{Ext}^{1}\left(\mathcal{E}_{n}, E\right) \longrightarrow \operatorname{Ext}^{1}\left(\mathcal{E}_{n-1}, E\right) \stackrel{\eta_{n}}{\longrightarrow} \operatorname{Ext}^{2}(E, E)=\mathbb{C} .
\end{aligned}
$$

On the other hand, the sequence (2) determines the non-zero element

$$
e_{n} \in \operatorname{Ext}^{1}\left(\mathcal{E}_{n-1}, E\right),
$$

and $\eta_{n}\left(e_{n}\right) \in \operatorname{Ext}^{2}(E, E)$ gives the obstruction to deforming $\left.\mathcal{E}\right|_{X \times S_{n}}$ to a coherent sheaf on $X \times S_{n+1}$ flat over $S_{n+1}$ (cf. [13, Prop. 3.13]). Therefore $\eta_{n}\left(e_{n}\right)=0$ for $n<m$ and $\eta_{m}\left(e_{m}\right) \neq 0$. On the other hand, we have the following morphism of exact sequences

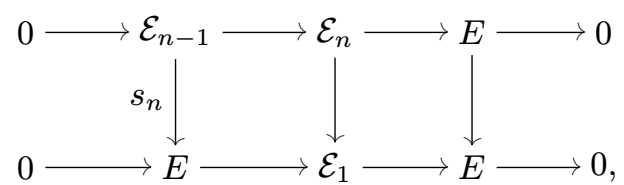

where $s_{n}$ is a natural surjection. Hence $\xi_{n}\left(s_{n}\right) \in \operatorname{Ext}^{1}(E, E)$ corresponds to the extension $\mathcal{E}_{1}$, which is a non-trivial first order deformation of $E$. Therefore $\xi_{n}\left(s_{n}\right) \neq 0$ and $\xi_{n}$ is surjective. Combining these, we have

$$
\begin{gathered}
\operatorname{Ext}^{1}\left(\mathcal{E}_{n-1}, E\right) \cong \operatorname{Ext}^{1}\left(\mathcal{E}_{n}, E\right) \cong \mathbb{C}(\text { for } n<m), \quad \operatorname{Ext}^{1}\left(\mathcal{E}_{m}, E\right) \cong 0 \\
\operatorname{Hom}\left(\mathcal{E}_{n-1}, E\right) \cong \operatorname{Hom}\left(\mathcal{E}_{n}, E\right) \cong \mathbb{C}
\end{gathered}
$$


Similarly applying $\operatorname{Hom}(E, *)$ to the sequence $(2)$, we obtain $\operatorname{Ext}^{1}\left(E, \mathcal{E}_{m}\right)=0$ and $\operatorname{Hom}\left(E, \mathcal{E}_{m}\right)=\mathbb{C}$. By Serre duality, we can conclude $\mathcal{E}$ is $R_{m}$-spherical.

Next let us consider the equivalence

$$
\widetilde{\Phi}:=T_{\mathcal{E}} \circ \Phi^{-1}: D(X) \longrightarrow D(X) .
$$

Then $\widetilde{\Phi}$ takes $\mathcal{O}_{C}(-1)$ to $\mathcal{O}_{C}(-1)$, and commutes with $\mathbb{R} f_{*}$. Therefore $\widetilde{\Phi}$ preserves perverse t-structure ${ }^{0} \operatorname{Per}(X / Y)$ in the sense of [3]. Then the argument of [14, Thm 6.1] shows $\widetilde{\Phi}$ is isomorphic to the identity functor.

\section{Deformations of $\mathbb{P}$-twists}

Review of $\mathbb{P}$-objects and associated twists. $-R$-spherical twists can also be used to construct deformations of $\mathbb{P}$-twists. Let us recall the definition of $\mathbb{P}$-objects and the associated autoequivalences introduced in [5]. Again we assume $X$ is a smooth projective variety over $\mathbb{C}$.

Definition 4.1 (see [5]). - An object $E \in D(X)$ is called $\mathbb{P}^{n}$-object if it satisfies the following:

- $\operatorname{Ext}_{X}^{*}(E, E)$ is isomorphic to $H^{*}\left(\mathbb{P}^{n}, \mathbb{C}\right)$ as a graded ring;

- $E \otimes \omega_{X} \cong E$.

Note that if $\mathbb{P}^{n}$-object exists, then $\operatorname{dim} X=2 n$ by Serre duality. D. Huybrechts and R. Thomas [5] constructed an equivalence $P_{E}: D(X) \rightarrow D(X)$ associated to $E$, which is described as follows. Let $h \in \operatorname{Ext}_{X}^{2}(E, E)$ be the degree two generator. First consider the morphism in $D(X \times X)$ :

$$
H:=\check{h} \otimes \mathrm{id}-\mathrm{id} \otimes h: \check{E} \otimes E[-2] \longrightarrow \check{E} \otimes E .
$$

Let us take its cone $\mathcal{H} \in D(X \times X)$. We can see the composition $H$ with the trace map tr: $\check{E} \otimes E \rightarrow \mathcal{O}_{\Delta}$ becomes zero. Therefore there exists a (in fact unique) morphism $t: \mathcal{H} \rightarrow \mathcal{O}_{\Delta}$ such that the following diagram commutes [5, Lemma 2.1]:

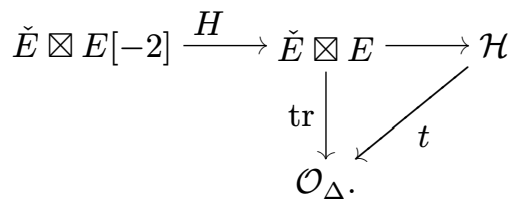

Then define $\mathcal{Q}_{\mathcal{E}}$ to be the cone

$$
\mathcal{Q}_{\mathcal{E}}:=\operatorname{Cone}\left(t: \mathcal{H} \rightarrow \mathcal{O}_{\Delta}\right) \in D(X \times X) .
$$

Then in [5], it is shown that the functor $P_{\mathcal{E}}: D(X) \rightarrow D(X)$ with kernel $\mathcal{Q}_{\mathcal{E}}$ gives the equivalence. 
Next let us consider a one parameter deformation of $X$. Let $f: \mathcal{X} \rightarrow C$ be a smooth family over a smooth curve $C$ with a distinguished fibre $j: X=$ $f^{-1}(0) \hookrightarrow \mathcal{X}, 0 \in C$. Suppose $E \in D(X)$ is a $\mathbb{P}^{n}$-object and let be its Atiyahclass $A(E) \in \operatorname{Ext}_{X}^{1}\left(E, E \otimes \Omega_{X}\right)$. Then the obstruction to deforming $E$ sideways to first order is given by the product

$$
A(E) \cdot \kappa(X) \in \operatorname{Ext}_{X}^{2}(E, E),
$$

where $\kappa(X) \in H^{1}\left(X, T_{X}\right)$ is the Kodaira-Spencer class of the family $f: \mathcal{X} \rightarrow C$. In [5], the case of $A(E) \cdot \kappa(X) \neq 0$ is studied. In that case, $j_{*} E$ is a spherical object and the associated equivalence $T_{j_{*} E}: D(\mathcal{X}) \rightarrow D(\mathcal{X})$ fits into the commutative diagram [5, Prop. 2.7]

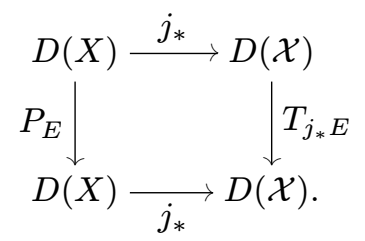

Our purpose is to treat the case of $A(E) \cdot \kappa(X)=0$.

$R$-spherical objects via deformations of $\mathbb{P}$-objects. - Let $f: \mathcal{X} \rightarrow C$ and $E \in$ $D(X)$ be as before, and assume $A(E) \cdot \kappa(X)=0$. Note that $j_{*} E$ is not spherical. In fact we have the distinguished triangle

$$
E[1] \longrightarrow \mathbb{L} j^{*} j_{*} E \longrightarrow E \stackrel{A(E) \cdot \kappa(X)}{\longrightarrow} E[2],
$$

by [5, Prop. 3.1]. Hence we have the decomposition $\mathbb{L} j^{*} j_{*} E \cong E \oplus E[1]$, and for $0 \leq k \leq 2 n+1$ we calculate

$$
\begin{aligned}
\operatorname{Ext}_{\mathcal{X}}^{k}\left(j_{*} E, j_{*} E\right) & \cong \operatorname{Ext}_{X}^{k}\left(\mathbb{L} j^{*} j_{*} E, E\right) \\
& \cong \operatorname{Ext}_{X}^{k}(E, E) \oplus \operatorname{Ext}_{X}^{k-1}(E, E) \cong \mathbb{C} .
\end{aligned}
$$

As in the previous section, we are going to consider deformations of $j_{*} E$ in $\mathcal{X}$. The moduli theories of complexes were carried out by $[6,9]$. Following the notation used in [6], we consider the functor $\operatorname{Splcpx}_{\mathcal{X} / C}$ from the category of locally noetherian schemes over $C$ to the category of sets,

$$
\begin{aligned}
& \operatorname{Splcpx}_{\mathcal{X} / C}(T) \\
& :=\left\{\begin{array}{l|l}
\mathcal{F}^{\bullet} & \begin{array}{l}
\mathcal{F}^{\bullet} \text { is a bounded complex of coherent sheaves on } \mathcal{X}_{T} \\
\text { such that each } \mathcal{F}^{i} \text { is flat over } T \text { and for any } t \in T, \\
\operatorname{Ext}_{X_{t}}^{0}\left(\mathcal{F}^{\bullet}(t), \mathcal{F}^{\bullet}(t)\right) \cong k(t), \operatorname{Ext}_{X_{t}}^{-1}\left(\mathcal{F}^{\bullet}(t), \mathcal{F}^{\bullet}(t)\right)=0
\end{array}
\end{array}\right\} \sim .
\end{aligned}
$$

Here

$$
\mathcal{X}_{T}:=\mathcal{X} \times_{C} T, \quad \mathcal{F}^{\bullet}(t):=\mathcal{F}^{\bullet} \otimes_{T} k(t),
$$


and $\mathcal{F}^{\bullet} \sim \mathcal{F}^{\bullet}$ if and only if there exist $\mathcal{L} \in \operatorname{Pic}(T)$, a bounded complex of quasi-coherent sheaves $\mathcal{G}^{\bullet}$ and quasi-isomorphisms $\mathcal{G}^{\bullet} \rightarrow \mathcal{F}^{\bullet}, \mathcal{G}^{\bullet} \rightarrow \mathcal{F}^{\bullet} \otimes \mathcal{L}$.

Let $\operatorname{Splcpx}_{\mathcal{X} / C}^{\text {ét }}$ be the associated sheaf of $\operatorname{Splcpx}_{\mathcal{X} / C}$ in the étale topology. M. Inaba [6] showed the following:

THEOREM 4.2 (see [6]). - The functor $\operatorname{Splcpx}_{\mathcal{X} / C}^{\text {ét }}$ is represented by a locally separated algebraic space $\mathcal{M}$ over $C$.

Let $S_{m}=\operatorname{Spec} \mathbb{C}[t] /\left(t^{m+1}\right)$ be as before and $\gamma: S_{1} \hookrightarrow C$ be an extension of $0 \hookrightarrow C$. Let $r$ be the restriction,

$$
r: \operatorname{Splcpx}_{\mathcal{X} / C}^{\text {ét }}(\gamma) \longrightarrow \operatorname{Splcpx}_{\mathcal{X} / C}^{\text {ét }}(0) .
$$

By the assumption $A(E) \cdot \kappa(X)=0$, we have $r^{-1}(E) \neq \varnothing$. Moreover by [6, Prop. 2.3], there is a bijection between $r^{-1}(E)$ and $\operatorname{Ext}_{X}^{1}(E, E)$, which is zero. Therefore the map $T_{\mathcal{M}, E} \rightarrow T_{C, 0}$ is an isomorphism, hence $\operatorname{dim} \mathcal{M} \leq 1$ at $[E] \in \mathcal{M}$. Note that by taking push-forward along the inclusion $\mathcal{X} \times{ }_{C} T \rightarrow \mathcal{X} \times T$, we get the morphism of functors:

$$
\delta: \operatorname{Splcpx}_{\mathcal{X} / C}^{\text {ét }} \longrightarrow \operatorname{Splcpx}_{\mathcal{X} / S_{0}}^{\text {ét }} .
$$

We put the following technical assumption:

The morphism $\delta$ gives an isomorphism between connected com$\left\{\right.$ ponents of both sides, which contain $E$ and $j_{*} E$ respectively. Let $[E] \in \mathcal{M}^{\prime} \subset \mathcal{M}$ be the connected component. We assume $\mathcal{M}^{\prime}$ is a zero-dimensional scheme.

Note that we can write $\mathcal{M}^{\prime}=S_{m}$ for some $m$. Let

$$
\mathcal{X}_{m}:=\mathcal{X} \times_{C} \mathcal{M}^{\prime}=\mathcal{X} \times_{C} S_{m}
$$

and $\mathcal{E} \in D\left(\mathcal{X}_{m}\right)$ be the universal family. We use the following notations for morphism:
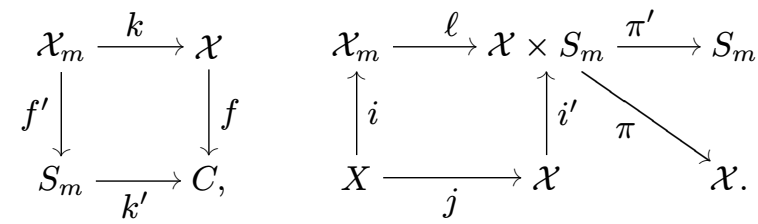

If there is no confusion, we will use the same notations for $n \leq m$. We show the following proposition:

Proposition 4.3. - The object $\ell_{*} \mathcal{E} \in D\left(\mathcal{X} \times S_{m}\right)$ is $R_{m}$-spherical. 
Proof. - Since $\pi_{*} \ell_{*} \mathcal{E} \cong k_{*} \mathcal{E}$ and $\mathbb{L} i^{\prime} \ell_{*} \mathcal{E} \cong j_{*} E$, we have to calculate $\operatorname{Ext}_{\mathcal{X}}^{i}\left(k_{*} \mathcal{E}, j_{*} E\right)$. By the assumption $(\star)$, we cannot deform $\ell_{*} \mathcal{E}$ to $(m+1)$-th order. For $n \leq m$, let $\mathcal{E}_{n}:=\left.\mathcal{E}\right|_{\mathcal{X}_{n}} \in D\left(\mathcal{X}_{n}\right)$ and $\widetilde{\mathcal{E}}_{n}:=k_{*} \mathcal{E}_{n} \in D(\mathcal{X})$. We consider distinguished triangles:

$$
\begin{aligned}
& \widetilde{\mathcal{E}}_{n-1} \longrightarrow \widetilde{\mathcal{E}}_{n} \longrightarrow j_{*} E \stackrel{e_{n}^{\prime}}{\longrightarrow} \widetilde{\mathcal{E}}_{n-1}[1], \\
& j_{*} E \longrightarrow \widetilde{\mathcal{E}}_{n} \longrightarrow \widetilde{\mathcal{E}}_{n-1} \stackrel{e_{n}}{\longrightarrow} j_{*} E[1] .
\end{aligned}
$$

Then by the argument of [13, Prop. 3.13], we can see that the composition

$$
e_{n} \circ e_{n}^{\prime}: j_{*} E \longrightarrow \widetilde{\mathcal{E}}_{n-1}[1] \longrightarrow j_{*} E[2]
$$

gives the obstruction to deforming $\ell_{*} \mathcal{E}_{n}$ to $(n+1)$-th order. If $E$ is a sheaf, this is just [13, Prop. 3.13] and we can generalize this by replacing the exact sequences in [13, Prop. 3.13] by the exact sequences of representing complexes. We leave the detail to the reader. Hence $e_{m} \circ e_{m}^{\prime} \neq 0$ and $e_{n} \circ e_{n}^{\prime}=0$ for $n<m$. Applying $\operatorname{Hom}\left(*, j_{*} E\right)$ to the triangle (3), we obtain the long exact sequence,

$$
\begin{aligned}
\operatorname{Ext}_{\mathcal{X}}^{1}\left(j_{*} E, j_{*} E\right) \longrightarrow & \operatorname{Ext}_{\mathcal{X}}^{1}\left(\widetilde{\mathcal{E}}_{n}, j_{*} E\right) \\
& \longrightarrow \operatorname{Ext}_{\mathcal{X}}^{1}\left(\widetilde{\mathcal{E}}_{n-1}, j_{*} E\right) \longrightarrow \operatorname{Ext}_{\mathcal{X}}^{2}\left(j_{*} E, j_{*} E\right)=\mathbb{C} .
\end{aligned}
$$

Then using the above sequence and the same argument as in Theorem 3.1, we can conclude $\operatorname{Ext}_{\mathcal{X}}^{1}\left(k_{*} \mathcal{E}, j_{*} E\right)=0$.

Next we use the existence of the distinguished triangle [1, Lemma 3.3]:

$$
\mathcal{E}[1] \longrightarrow \mathbb{L} k^{*} k_{*} \mathcal{E} \longrightarrow \mathcal{E} \longrightarrow \mathcal{E}[2] .
$$

Pulling back to $X$, we have the triangle

$$
E[1] \longrightarrow \mathbb{L} j^{*} k_{*} \mathcal{E} \longrightarrow E \stackrel{\theta}{\longrightarrow} E[2] .
$$

Since $\operatorname{Ext}_{X}^{2}(E, E)$ is one dimensional, $\theta$ is zero or non-zero multiple of $h$. Assume $\theta=0$. Then $\mathbb{L} j^{*} k_{*} \mathcal{E} \cong E \oplus E[1]$, and

$$
\begin{aligned}
\operatorname{Ext}_{\mathcal{X}}^{1}\left(k_{*} \mathcal{E}, j_{*} E\right) & \cong \operatorname{Ext}_{\mathcal{X}}^{1}\left(\mathbb{L} j^{*} k_{*} \mathcal{E}, E\right) \\
& \cong \operatorname{Ext}_{X}^{1}(E, E) \oplus \operatorname{Hom}(E, E) \cong \mathbb{C},
\end{aligned}
$$

which is a contradiction. Hence we may assume $\theta=h$. Applying $\operatorname{Hom}(*, E)$ to the triangle (5), we obtain the long exact sequence

$$
\rightarrow \operatorname{Ext}_{X}^{i}(E, E) \longrightarrow \operatorname{Ext}_{\mathcal{X}}^{i}\left(\mathbb{L} j^{*} k_{*} \mathcal{E}, E\right) \longrightarrow \operatorname{Ext}_{X}^{i-1}(E, E) \stackrel{h}{\longrightarrow} \operatorname{Ext}_{X}^{i+1}(E, E) \rightarrow \text {. }
$$

By the definition of $\mathbb{P}^{n}$-object, we obtain

$$
\operatorname{Ext}_{\mathcal{X}}^{i}\left(k_{*} \mathcal{E}, j_{*} E\right) \cong \operatorname{Ext}_{X}^{i}\left(\mathbb{L} j^{*} k_{*} \mathcal{E}, E\right)= \begin{cases}\mathbb{C} & \text { if } i=0 \text { or } i=2 n+1, \\ 0 & \text { otherwise. }\end{cases}
$$


Remark 4.4. - Assumption $(\star)$ is satisfied if $E$ is a sheaf and $\operatorname{dim} \mathcal{M}^{\prime}=0$. In fact suppose $\ell_{*} \mathcal{E}$ extends to a $S_{m+1}$-valued point of $\operatorname{Splcpx}_{\mathcal{X} / S_{0}}^{\text {ét }}$. Then as in [13, Prop. 3.13], there exists $\widetilde{\mathcal{E}}_{m+1} \in \operatorname{Coh}(\mathcal{X})$ such that there exists a morphism of exact sequences of $\mathcal{O}_{\mathcal{X}}$-modules:

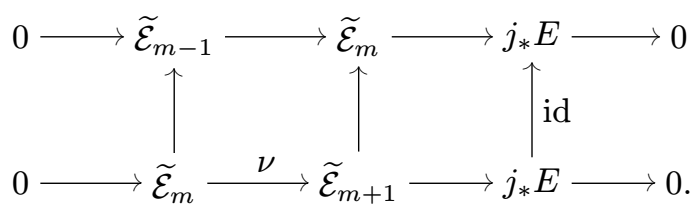

An easy diagram chase shows $\widetilde{\mathcal{E}}_{m+1}$ is a $\mathcal{O}_{\mathcal{X}} /\left(t^{m+2}\right)$-module for the uniformizing parameter $t \in \mathcal{O}_{C, 0}$. Moreover we have $t \cdot \widetilde{\mathcal{E}}_{m+1}=\operatorname{Im} \nu$. Therefore the map

$$
\widetilde{\mathcal{E}}_{m+1} \otimes_{\mathcal{O}_{C} /\left(t^{m+2}\right)}(t) \longrightarrow \widetilde{\mathcal{E}}_{m+1}
$$

is a morphism from $\widetilde{\mathcal{E}}_{m}$ onto $\operatorname{Im} \nu \cong \widetilde{\mathcal{E}}_{m}$, hence injective. Then [13, Lemma 3.7] shows $\widetilde{\mathcal{E}}_{m+1}$ is flat over $\mathcal{O}_{C, 0} /\left(t^{m+2}\right)$ and gives a $S_{m+1}$-valued point of Splcpx ${ }_{\mathcal{X} / C}^{\text {et }}$.

$\mathbb{P}$-twists and $R$-spherical twists. - By Proposition 4.3, we have the associated functor $T_{\ell_{*} \mathcal{E}}: D(\mathcal{X}) \rightarrow D(\mathcal{X})$ under assumption $(\star)$. The next purpose is to show the existence of the diagram as in [5, Prop. 2.7]. We use the following notations for morphisms:
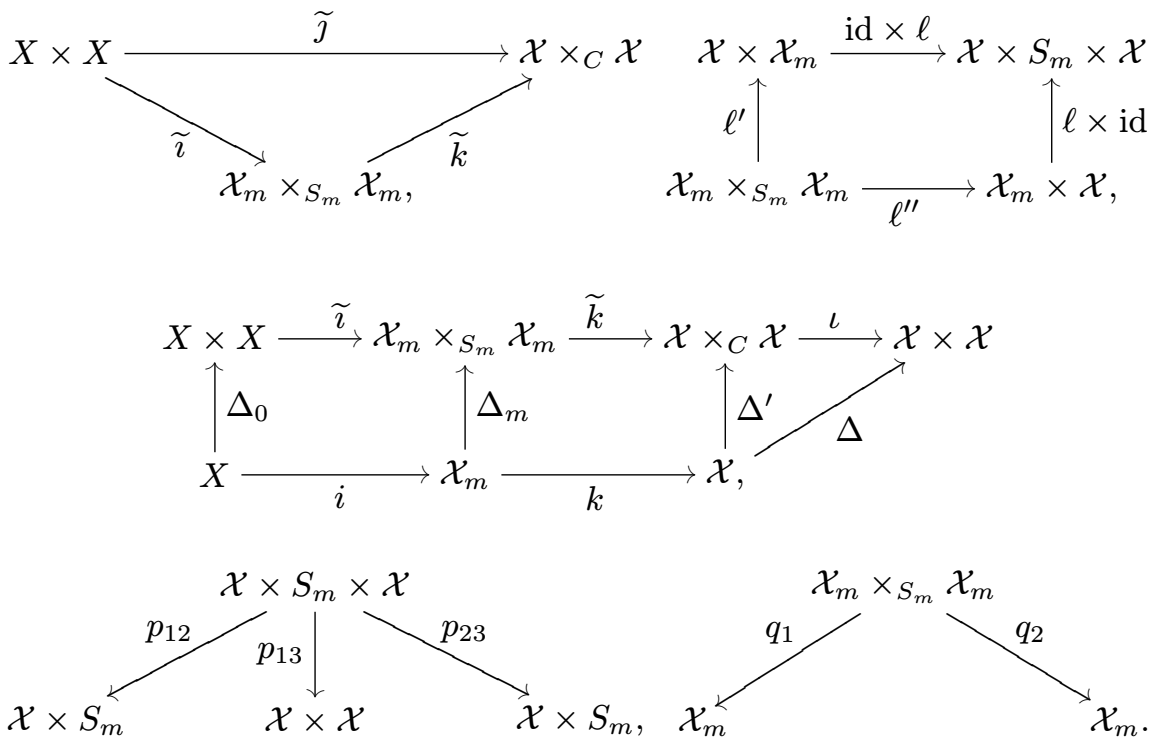
TheOREM 4.5. - The functor $T_{\ell_{*} \mathcal{E}}$ fits into the following commutative diagram:

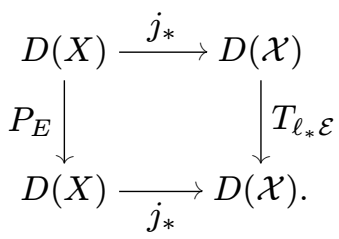

Proof. - We try to imitate the argument of [5, Prop. 2.7]. First we construct the morphism

$$
\alpha: \widetilde{k}_{*}\left(q_{1}^{*} \check{\mathcal{E}} \stackrel{\mathbb{L}}{\otimes} q_{2}^{*} \mathcal{E}\right)[-1] \longrightarrow \Delta_{*}^{\prime} \mathcal{O}_{\mathcal{X}}
$$

in $D\left(\mathcal{X} \times_{C} \mathcal{X}\right)$. This is constructed by the composition of $\widetilde{k}_{*} \operatorname{tr}$,

$$
\widetilde{k}_{*} \operatorname{tr}: \widetilde{k}_{*}\left(q_{1}^{*} \check{\mathcal{E}} \stackrel{\mathbb{L}}{\otimes} q_{2}^{*} \mathcal{E}\right)[-1] \longrightarrow \widetilde{k}_{*} \Delta_{m *} \mathcal{O}_{\mathcal{X}_{m}}[-1]=\Delta_{*}^{\prime} k_{*} \mathcal{O}_{\mathcal{X}_{m}}[-1],
$$

with the morphism $\Delta_{*}^{\prime} k_{*} \mathcal{O}_{\mathcal{X}_{m}}[-1] \rightarrow \Delta_{*}^{\prime} \mathcal{O}_{\mathcal{X}}$ obtained by applying $\Delta_{*}^{\prime}$ to the exact sequence,

$$
0 \longrightarrow \mathcal{O}_{\mathcal{X}} \longrightarrow \mathcal{O}_{\mathcal{X}}\left(\mathcal{X}_{m}\right) \longrightarrow k_{*} \mathcal{O}_{\mathcal{X}_{m}} \longrightarrow 0
$$

Let $\mathcal{L}:=\operatorname{Cone}(\alpha) \in D\left(\mathcal{X} \times_{C} \mathcal{X}\right)$. Applying Chen's lemma [4], it suffices to show

$$
\iota_{*} \mathcal{L} \cong \operatorname{Cone}\left(\mathbb{R} p_{13 *}\left(p_{12}^{*}\left(\ell_{*}^{\circ} \mathcal{E} \stackrel{\mathbb{L}}{\otimes} \pi^{!} \mathcal{O}_{\mathcal{X}}\right) \stackrel{\mathbb{L}}{\otimes} p_{23}^{*} \ell_{*} \mathcal{E}\right) \stackrel{\mu}{\longrightarrow} \Delta_{*} \mathcal{O}_{\mathcal{X}}\right), \quad \mathbb{L} \widetilde{\jmath}^{*} \mathcal{L} \cong \mathcal{H}
$$

Here $\mu$ is the morphism constructed in the proof of Theorem 2.3 and $\mathcal{H}$ is the kernel of $P_{E}$. First we check $\iota_{*} \mathcal{L} \cong \operatorname{Cone}(\mu)$. Note that $\pi^{!} \mathcal{O}_{\mathcal{X}}=\mathcal{O}_{\mathcal{X} \times S_{m}}$ and $\ell_{*} \mathcal{E} \cong \ell_{*} \check{\mathcal{E}}[-1]$ by the duality isomorphism. Hence we have

$$
\begin{gathered}
\mathbb{R} p_{13 *}\left(p_{12}^{*}\left(\ell_{*} \mathcal{E} \stackrel{\mathbb{L}}{\otimes} \pi^{!} \mathcal{O}_{\mathcal{X}}\right) \stackrel{\mathbb{L}}{\otimes} p_{23}^{*} \ell_{*} \mathcal{E}\right) \cong \mathbb{R} p_{13 *}\left(p_{12}^{*} \ell_{*} \check{\mathcal{E}} \stackrel{\mathbb{L}}{\otimes} p_{23}^{*} \ell_{*} \mathcal{E}\right)[-1] \\
\cong \mathbb{R} p_{13 *}\left\{(\ell \times \mathrm{id})_{*} r_{1}^{*} \check{\mathcal{E}} \stackrel{\mathbb{L}}{\otimes}(\mathrm{id} \times \ell)_{*} r_{2}^{*} \mathcal{E}\right\}[-1] \\
\cong \mathbb{R} p_{13 *}(\mathrm{id} \times \ell)_{*}\left\{\mathbb{L}(\mathrm{id} \times \ell)^{*}(\ell \times \mathrm{id})_{*} r_{1}^{*} \check{\mathcal{E}} \otimes r_{2}^{*} \mathcal{E}\right\}[-1] \\
\cong \mathbb{R} p_{13 *}(\mathrm{id} \times \ell)_{*}\left(\ell_{*}^{\prime} \mathbb{L} \ell^{\prime \prime} r_{1}^{*} \check{\mathcal{E}} \stackrel{\mathbb{L}}{\otimes} r_{2}^{*} \mathcal{E}\right)[-1] \\
\cong \mathbb{R} p_{13 *}(\mathrm{id} \times \ell)_{*} \ell_{*}^{\prime}\left(\mathbb{L} \ell^{\prime \prime} r_{1}^{*} \check{\mathcal{E}} \stackrel{\mathbb{L}}{\otimes} \mathbb{L} \ell^{*} r_{2}^{*} \mathcal{E}\right)[-1] \\
\cong \iota_{*} \widetilde{k}_{*}\left(q_{1}^{*} \check{\mathcal{E}} \stackrel{\mathbb{L}}{\otimes} q_{2}^{*} \mathcal{E}\right)[-1] .
\end{gathered}
$$


Here $r_{1}, r_{2}$ are defined by the fiber squares:
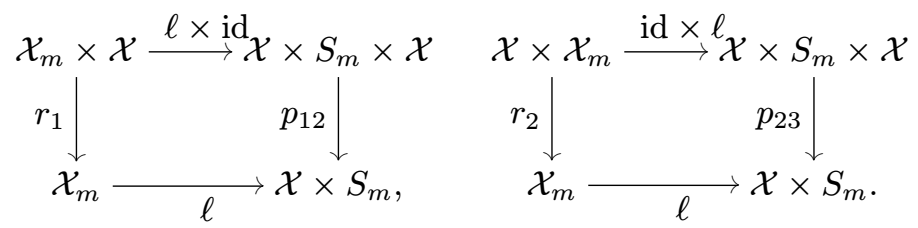

Under the above isomorphism, we can check $\iota_{*} \alpha=\mu$. Hence $\widetilde{\ell}_{*} \mathcal{L} \cong \operatorname{Cone}(\mu)$. Next we check $\mathbb{L} \tilde{\jmath}^{*} \mathcal{L} \cong \mathcal{H}$. Note that we have

$$
\mathbb{L} \widetilde{\jmath}^{*} \mathcal{L}=\operatorname{Cone}\left(\mathbb{L} \widetilde{\jmath}^{*} \widetilde{k}_{*}\left(q_{1}^{*} \check{\mathcal{E}} \stackrel{\mathbb{L}}{\otimes} q_{2}^{*} \mathcal{E}\right)[-1] \stackrel{\mathbb{L} \widetilde{\jmath}^{*} \alpha}{\longrightarrow} \mathbb{L} \widetilde{\jmath}^{*} \Delta_{*}^{\prime} \mathcal{O}_{\mathcal{X}}=\Delta_{0 *} \mathcal{O}_{X}\right),
$$

and there exists the distinguished triangle

$$
q_{1}^{*} \check{\mathcal{E}} \stackrel{\mathbb{L}}{\otimes} q_{2}^{*} \mathcal{E}[-2] \longrightarrow q_{1}^{*} \check{\mathcal{E}} \stackrel{\mathbb{L}}{\otimes} q_{2}^{*} \mathcal{E} \longrightarrow \mathbb{L} \widetilde{k}^{*} \widetilde{k}_{*}\left(q_{1}^{*} \check{\mathcal{E}} \stackrel{\mathbb{L}}{\otimes} q_{2}^{*} \mathcal{E}\right)[-1]
$$

as in [1, Lemma 3.3$]$. Then applying $\mathbb{L} \widetilde{\imath}^{*}$, we have the triangle

$$
\check{E} \otimes E[-2] \stackrel{u}{\longrightarrow} \check{E} \otimes E \longrightarrow \mathbb{L} \widetilde{\jmath}^{*} \widetilde{k}_{*}\left(q_{1}^{*} \check{\mathcal{E}} \stackrel{\mathbb{L}}{\otimes} q_{2}^{*} \mathcal{E}\right)[-1] .
$$

We can easily check the following:

$$
\begin{aligned}
& \operatorname{Ext}_{X \times X}^{2}(\check{E} \otimes E, \check{E} \otimes E) \\
& \quad \cong\left(\operatorname{Ext}_{X}^{2}(E, E) \otimes \operatorname{Ext}_{X}^{0}(E, E)\right) \oplus\left(\operatorname{Ext}_{X}^{0}(E, E) \otimes \operatorname{Ext}_{X}^{2}(E, E)\right) .
\end{aligned}
$$

Hence we can write $u=a(\check{h} \otimes \mathrm{id})+b(\mathrm{id} \otimes h)$ for some $a, b \in \mathbb{C}$. On the other hand, we can check that the following diagram commutes:

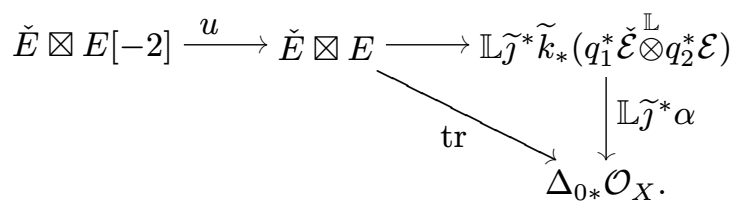

This is easily checked using the same argument of [5, Prop. 2.7], and leave the detail to the reader. Therefore $\operatorname{tr} \circ u=0$, which implies $b=-a$. Hence if we show $u \neq 0$, then we can conclude $\mathbb{L} \widetilde{\jmath}^{*} \mathcal{L} \cong \mathcal{H}$. Assume $u=0$. Then we have the decomposition

$$
\mathbb{L} \widetilde{\jmath}^{*} \widetilde{k}_{*}\left(q_{1}^{*} \check{\mathcal{E}} \stackrel{\mathbb{L}}{\otimes} q_{2}^{*} \mathcal{E}\right)[-1] \cong(\check{E} \otimes E) \oplus(\check{E} \otimes E)[-1] .
$$

Since $\operatorname{Hom}_{X \times X}\left(\check{E} \otimes E[-1], \Delta_{*} \mathcal{O}_{X}\right)=0$, the morphism

$$
\mathbb{L} \widetilde{\jmath}^{*} \alpha: \mathbb{L} \widetilde{\jmath}^{*} \widetilde{k}_{*}\left(q_{1}^{*} \check{\mathcal{E}} \stackrel{\mathbb{L}}{\otimes} q_{2}^{*} \mathcal{E}\right)[-1] \longrightarrow \Delta_{0 *} \mathcal{O}_{X}
$$


is a non-zero multiple of ( $\operatorname{tr}, 0)$ under the decomposition (6). Let $\mathcal{S} \in D(X \times X)$ be the cone of the trace map:

$$
\check{E} \otimes E \stackrel{\operatorname{tr}}{\longrightarrow} \Delta_{0 *} \mathcal{O}_{X} \longrightarrow \mathcal{S} .
$$

Then we have the decomposition $\mathbb{L} \widetilde{\jmath}^{*} \mathcal{L} \cong \mathcal{S} \oplus(\check{E} \otimes E)$, and the following diagram commutes by Chen's lemma [4]:

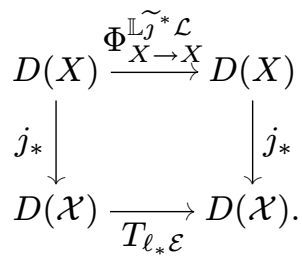

In particular we have

$$
j_{*} \Phi_{X \rightarrow X}^{\stackrel{\mathbb{L}^{*} \mathcal{L}}{L}}(E) \cong T_{\ell_{*} \mathcal{E}}\left(j_{*} E\right) \cong j_{*} E[1-\operatorname{dim} \mathcal{X}],
$$

which is indecomposable. It follows that

$$
\Phi_{X \rightarrow X}^{\mathcal{S}}(E) \cong 0 \quad \text { or } \quad \Phi_{X \rightarrow X}^{\check{E} \otimes E}(E) \cong 0 .
$$

Since $\Phi_{X \rightarrow X}^{\check{E} \otimes E}(E) \cong \mathbb{R} \operatorname{Hom}(E, E) \otimes_{\mathbb{C}} E$, the latter is impossible by the definition of $\mathbb{P}^{n}$-object. Hence $\Phi_{X \rightarrow X}^{\mathcal{S}}(E)$ must be zero. Since we have the distinguished triangle:

$$
\mathbb{R} \operatorname{Hom}(E, E) \otimes_{\mathbb{C}} E \longrightarrow E \longrightarrow \Phi_{X \rightarrow X}^{\mathcal{S}}(E) \cong 0,
$$

we have $\mathbb{R} \operatorname{Hom}(E, E) \otimes_{\mathbb{C}} E \cong E$. But again this is impossible by the definition of $\mathbb{P}^{n}$-object.

\section{BIBLIOGRAPHY}

[1] A. Bondal \& D. ORLOV - "Semiorthogonal decomposition for algebraic varieties", preprint, 1995, arXiv:math.AG/9506012, p. 1-55.

[2] T. BRIDGELAND - "Equivalences of triangulated categories and FourierMukai transforms", Bull. London Math. Soc. 31 (1999), p. 25-34.

[3] _ _ "Flops and derived categories", Invent. Math. 147 (2002), p. 613632.

[4] J.-C. Chen - "Flops and equivalences of derived categories for threefolds with only Gorenstein singularities", J. Differential Geom. 61 (2002), p. 227-261.

[5] D. Huybrechts \& R. Thomas - "P-objects and autoequivalences of derived categories", preprint, 2005, arXiv:math.AG/0507040, p. 1-13. 
[6] M. INABA - "Toward a definition of moduli of complexes of coherent sheaves on a projective scheme", J. Math. Kyoto Univ. 42-2 (2002), p. 317329 .

[7] A. IshiI \& H. Uehara - "Autoequivalences of derived categories on the minimal resolutions of $A_{n}$-singularities on surfaces", preprint, 2004, arXiv:math.AG/0409151, p. 1-53.

[8] M. Kontsevich - "Homological algebra of mirror symmetry", in Proceedings of the International Congress of Mathematicians, Zurich (1994) vol. I, Birkhäuser, 1995, p. 120-139.

[9] M. Lieblich - "Moduli of complexes on a proper morphism", J. Algebraic Geom. 15 (2006), p. 175-206.

[10] D. Ploog - "Autoequivalences of derived categories of smooth projective varieties", Ph.D. Thesis, 2005.

[11] P. SEIDEL - "Graded Lagrangian submanifolds", Bull. Soc. Math. France 128 (2000), p. 103-149.

[12] P. Seidel \& R. Thomas - "Braid group actions on derived categories of coherent sheaves", Duke Math. J. 108 (2001), p. 37-107.

[13] R. Thomas - "A holomorphic casson invariant for Calabi-Yau 3-folds and bundles on K3-fibrations", J. Differential Geom. 54 (2000), p. 367-438.

[14] Y. TODA - "Stability conditions and crepant small resolutions", preprint, 2005, arXiv:math.AG/0512648, p. 1-25. 